\title{
PALINOLOGIA DE LA TRANSICIÓN CAMBRO-ORDOVÍCICA EN QUEBRADA DE MOYA, CORDILLERA ORIENTAL, ARGENTINA
}

\author{
LUCÍA ARÁOZ \\ CONICET, Universidad Nacional de Tucumán Facultad de Ciencias Naturales e I.M.L. Miguel Lillo, 205, (4000) San Miguel de \\ Tucumán, Argentina.lucia_araoz@yahoo.com.ar \\ MARÍA DEL MILAGRO VERGEL \\ CONICET, Universidad Nacional de Tucumán, Facultad de Ciencias Naturales e I.M.L. Miguel Lillo, 251 (4000) San Miguel de \\ Tucumán, Argentina.vergelmar@tucbbs.com.ar
}

\begin{abstract}
RESUMO - Este trabalho documenta a presença de acritarcos e formas relacionadas nas formações Casa Colorada e Alfarcito, aflorantes na Passagem de Moya, província de Jujuy, noroeste da Argentina. A composição taxonômica e a distribuição estratigráfica dos táxons permitiram diferenciar três associações palinológicas. A primeira, localizada na seção inferior da Formação Casa Colorada, apresenta baixa diversidade e má preservação. Contudo, contem esferomorfos simples (Leiosphaeridia, Lophosphaeridium, Saharidia) indicadores de condições marinhas pouco profundas, corroborando interpretações dos dados sedimentológicos. A segunda associação foi recuperada na seção média da Formação Casa Colorada e inclui uma microflora mais diversificada, com Vulcanisphaera africana, Cristallinium cambriense e Cymatiogalea velifera, entre outras, que permitiu posicionamento, juntamente com a primeira associação, no Cambriano final. A terceira associação, presente na porção superior da Formação Casa Colorada e na Formação Altarcito, contém várias espécies que indicam uma idade tremadociana, dentre as quais Buedingiisphaeridium tremadocum, Cymatiogalea membranispina e Polygonium symbolum. Os dados palinológicos sugerem uma idade cambriana final para os dois conjuntos inferiores (porções inferior e média da Formação Casa Colorada), e ordoviciana inicial para o conjunto superior (porção superior da Formação Casa Colorada e Formação Alfarcito), corroborando dados da fauna fóssil. Na Passagem de Moya, o limite entre os sistemas Cambriano e Ordoviciano é inferido como correspondente à parte mais alta da Formação Casa Colorada.
\end{abstract}

Palabras-chave: Palinologia, acritarcos, Cambro-ordoviciano, Passagem de Moya, Argentina

ABSTRACT - PALYNOLOGY OF THE CAMBRO-ORDOVICIAN TRANSITION AT QUEBRADA DE MOYA, EASTERN CORDILLERA, ARGENTINA. This contribution documents the presence of acritarchs and related forms in the Casa Colorada and Alfarcito formations cropping out at the Quebrada de Moya in the Quebrada de Humahuaca, Jujuy province, Northwest Argentina. The taxonomic composition and the biostratigraphic distribution of taxa allows to define three palynological assemblages. The first is located in the lower part of the Casa Colorada Formation and records a restricted diversity with poor preservation. However, it contains simple spheromorphs (Leiosphaeridia, Lophosphaeridium, Saharidia), indicating a shallow-marine conditions, coincident with the sedimentological characterization of strata. The following assemblage was recovered from the middle section of the Casa Colorada Formation and includes a more diversified microflora with Vulcanisphaera africana, Cristallinium cambriense, Cymatiogalea velifera, among others, that have been assigned with the early mentioned assemblage to the Upper Cambrian. The third assemblage was located in the upper sector of the Casa Colorada Formation and the Alfarcito Formation, and comprises several species, among which Buedingiisphaeridium tremadocum, Cymatiogalea membranispina, Polygonium symbolum are indicative of a Tremadocian age. Palynological data suggests an Upper Cambrian age for the two lower associations (lower and middle sector of the Casa Colorada Formation), and an Early Ordovician age for the upper association (uppermost sector of the Casa Colorada and Alfarcito formations), in concordance with other shelly fauna mentioned for the units. The position of the boundary between the Cambrian and Ordovician System in the Quebrada de Moya is inferred in the uppermost part of the Casa Colorada Formation.

Key words: Palynology, acritarchs, Cambrian-Ordovician, Moya Creek, Argentina. 


\section{INTRODUCCIÓN}

La Quebrada de Moya se halla ubicada en el margen oriental del Río Grande, $10 \mathrm{~km}$ al sudeste de la ciudad de Humahuaca (provincia de Jujuy), Cordillera Oriental, Argentina (Figura 1). La misma corresponde a una de las clásicas secciones en las que aflora el Grupo Santa Victoria (Turner, 1960), unidad litoestratigráfica siliciclástica cuya fauna de invertebrados fósiles registra el pasaje entre el Cámbrico y el Ordovícico (Moya, 1988; Aceñolaza \& Albanesi, 1997; Di Cunzolo et al., 2003). El Grupo Santa Victoria ha sido analizado en sus diferentes aspectos sedimentológicos, es así que Moya $(1988 ; 1999)$ y Buatois \& Mángano (2003) lo interpretan como un complejo marino que evoluciona desde ambientes restringidos fluviales y estuarinos a ambientes marinos abiertos.

En Quebrada de Moya, el Grupo Santa Victoria sobreyace en contacto discordante al Grupo Mesón (Cámbrico). Este contacto ha sido objeto de variables interpretaciones, según ciertos autores (e.g., Turner, 1960; Moya, 1988) es el resultado de los movimientos tectónicos de la Orogenia Irúyica; para otros fue producida por una relativa caída del nivel del mar (Aceñolaza, 1996; Buatois \& Mángano, 2003; Moya, 1998, 1999, 2002). Estudios recientes que se vienen desarrollando sobre las unidades antes mencionadas sugieren un origen dual tectono-eustático para el evento Irúyico. Hacia arriba, estratos cuaternarios de la Formación Uquía se disponen discordantemente sobre la sucesión cambro-ordovícica.

La complejidad litológica de los depósitos cambroordovícicos del norte argentino ha generado una profusión de nominaciones de diferente categoría según el área en que ocurren. Es así que algunos autores designan los cuerpos litológicos de la comarca de Humahuaca informalmente (Harrington, 1957), con categoría formacional (López \& Nullo, 1969; Moya, 1988, 1999; Aceñolaza \& Poiré, 1999; Zeballo et al., 2003), o con carácter de miembros (Buatois \& Mángano, 2003). En este trabajo adoptamos la propuesta de López \& Nullo (1969) seguida por Aceñolaza \& Poiré (1999). Según este esquema, la unidad basal del Grupo Santa Victoria corresponde a la Formación Casa Colorada, la misma representa una sucesión arenoso-pelítica de tonos verdes, grises y negros donde se diferencian dos miembros: uno inferior arenoso-cuarcítico, caracterizado por sedimentación mareal y estuarina (Moya, 1997; Buatois \& Mángano, 2003), denominado por estos últimos autores como Miembro Tilcara. Un miembro superior pelítico, en el esquema de López \& Nullo (1969), que representa la transición shoreface/offshore inferior y grada en su parte cuspidal a niveles heterolíticos de un sistema dominado por oleaje (Aceñolaza, 1996; Di Cunzolo et al., 2003; Buatois \& Mángano, 2003; Aceñolaza et al., 2003), equivalente al Miembro Casa Colorada según la terminología de Buatois \& Mángano (2003).

La Formación Casa Colorada que presenta una potencia aproximada de $150 \mathrm{~m}$ en el área analizada, registra niveles con trazas fósiles que según Aceñolaza (1996) corresponden a una biota adaptada a facies de alta y moderada energía, en partes oportunistas, dominadas por elementos suspensívoros en sus sectores arenoso-cuarcíticos. Los elementos bentónicos detritívoros (e.g., infaunales) son los más frecuentes en la base de los niveles tempestíticos, y aquellas facies heterolíticas están comunmente representadas en los niveles de transición hacia la unidad superior.

Diferentes grupos fósiles complementan la información paleontológica de la unidad, trilobites, braquiópodos inarticulados, equinodermos etc., que se desarrollan dentro de la Biozona de Parabolina (N.) frequens argentina (Trilobita). En contacto transicional sobreyacen areniscas escasamente fosilíferas, con conspicuas estructuras sedimentarias hummocky y estratificación entrecruzada, corresponden a la Formación Alfarcito (Tremadociano). La sucesión se continúa transicionalmente con las pelitas y areniscas fosilíferas de la Formación Rupasca (Tremadociano superior) con trilobites de la Biozona Bienvillia tetragonalis-Conophrys minutula.

No obstante la presencia de diferentes grupos fósiles que indican una segura antigüedad entre el Cámbrico Tardío y el Tremadociano, en general el Grupo Santa Victoria no tiene establecido un nivel litológico para la transición entre ambos sistemas. Recientemente, estudios palinológicos realizados en Quebrada de Moya por Aceñolaza et al. (2003) registran en los niveles cuspidales de la Formación Casa Colorada o miembro pelítico superior, una asociación palinológica asignada al Tremadociano más temprano. Al mismo tiempo, Rubinstein et al. (2003) reconocen dentro de la misma sección, en la porción basal del miembro pelítico o base del Miembro Casa Colorada (esquema de Buatois \& Mángano, 2003), una asociación cámbrica tardía de acritarcos y prasinofitas.

Al registrar microfloras algo disímiles en el mismo perfil estratigráfico, es que se realizó un detallado muestreo de la sección a fin de probar otros horizontes fértiles y poder delimitar una

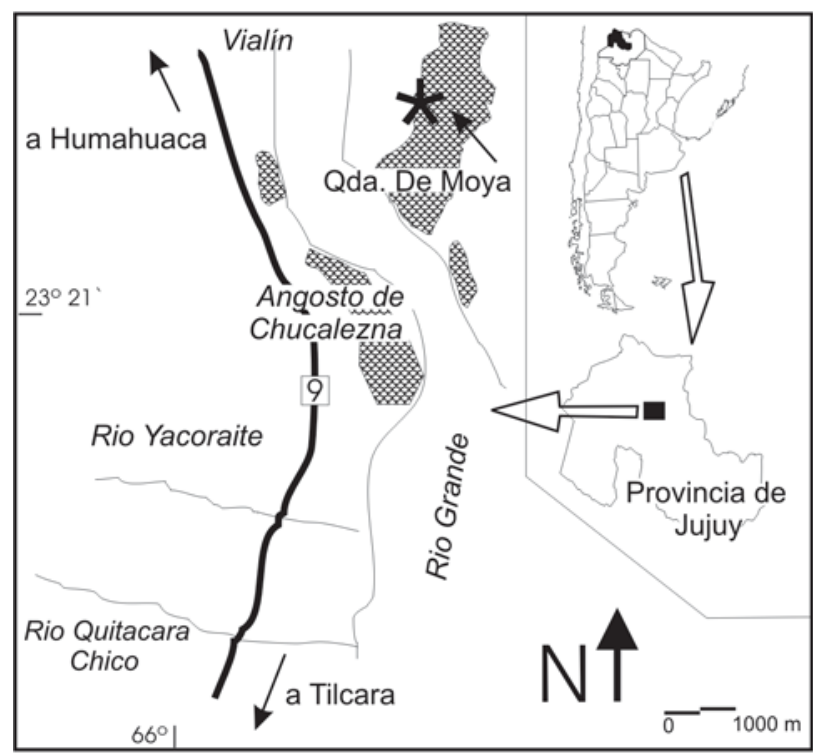

Figura 1. Mapa de ubicación de la Quebrada de Moya mostrando las areas con afloramientos cambro-ordovicianos y sitio de coleta (asterisco)(modificado de Aceñolaza et al., 2003).

Figure 1. Location map of Quebrada de Moya showing the cambroordovician outcrop areas (shaded) and collecting site (asterisk) (modified from Aceñolaza et al., 2003). 
ubicación más precisa del nivel litológico que registra el pasaje entre el Cámbrico y el Ordovícico, sobre todo considerando que la distancia entre las asociaciones mencionadas registran una separación de aproximadamente $60 \mathrm{~m}$.

\section{MATERIALES Y MÉTODOS}

La sección de Quebrada de Moya fue muestreada en toda su extensión y entre los niveles recolectados, sólo seis brindaron material fértil. Los mismos se distribuyen más o menos equidistantes entre las Formaciones Casa Colorada y Alfarcito (Figura 2). Se procesaron en el Laboratorio Paleontológico del Departamento Paleontología de la Fundación Miguel Lillo, siguiendo técnicas usuales de maceración y separación ( $\mathrm{HCl}, \mathrm{HF})$, sin uso de centrífuga ni oxidación. Los residuos fueron separados con mallas de 20 $\mu \mathrm{m}$ y el montaje realizado en glicerina gelatina. Los preparados palinológicos están depositados en la Colección Paleobotánica (preparados Microscópicos) del Instituto Miguel Lillo, Tucumán, Argentina, con la sigla y números LIL PB-PM 491 - 496.

El material se observó en microscopio de luz transmitida y los especimenes fotografiados registran las coordenadas del England Finder.

\section{RESULTADOS PALINOLÓGICOS}

El análisis taxonómico de las muestras permitió diferenciar una microflora integrada por 43 especies de palinomorfos, distribuidas en tres asociaciones con diferencias tanto cualitativas como cuantitativas (Figuras 3-6). En el sector medio de la Formación Casa Colorada (parcialmente equivalente al miembro Tilcara de Buatois \& Mángano (2003), se registran dos niveles de muestreo, muestras 6 (LIL PB-PM-491) y 5 (LIL PBPM-492) en Figura 2, que determinan una asociación caracterizada por escasa abundancia y diversidad como también pobre preservación, con un alto índice de alteración térmica que dificultó en muchos casos la determinación sistemática a nivel específico. Está mayormente constituida por formas esferoidales simples como Lophosphaeridium spp. y Leiosphaeridia spp.; también Leiofusa sp., Ooidium rossicum, Veryhachium sp., Timofeevia phosphoritica y cf. Orthosphaeridium? extensum como constituyentes menores. A excepción de las especies Ooidium rossicum y cf. Orthosphaeridium? extensum que son asignadas al Cámbrico Tardío, la asociación no presenta suficientes formas de valor bioestratigráfico, no obstante le asignamos dicha antigüedad, tomando como otro parámetro de control, la microflora asignada al Cámbrico Tardío por Rubinstein et al. (2003), recuperada de niveles próximos superiores. Además de acuerdo a lo establecido por Vecoli \& Le Hérissé (2004) para numerosas asociaciones cambro-ordovícicas del Margen Norte de Gondwana, en proximidad del límite sistémico, se destaca una baja diversidad y un neto incremento en la abundancia de leiosferas, las cuales pueden ser dominantes o exclusivas de las asociaciones. La presencia de estos tipos esferomorfos permitió también realizar inferencias paleoambientales para los niveles portadores. Como señalan varios autores (Tongiorgi et al., 1998;
Vecoli, 2000; entre otros) los géneros incluidos dentro del grupo informal esferomorfitas, como Leiosphaeridia, Lophosphaeridium, Saharidia, caracterizados por la presencia de una vesícula de pared delgada y simple, son particularmente abundantes en las capas superiores de mares someros y restringidas a ambientes cercanos a la playa, datos que coinciden con las interpretaciones sedimentológicas y faunísticas.

Los dos niveles siguientes del muestreo ubicados en el sector medio a superior pelítico de la Formación Casa Colorada, muestras 4 (LIL PB-PM-493) y 3 (LIL PB-PM-494) en Figura 2, brindaron una asociación palinológica más diversificada y

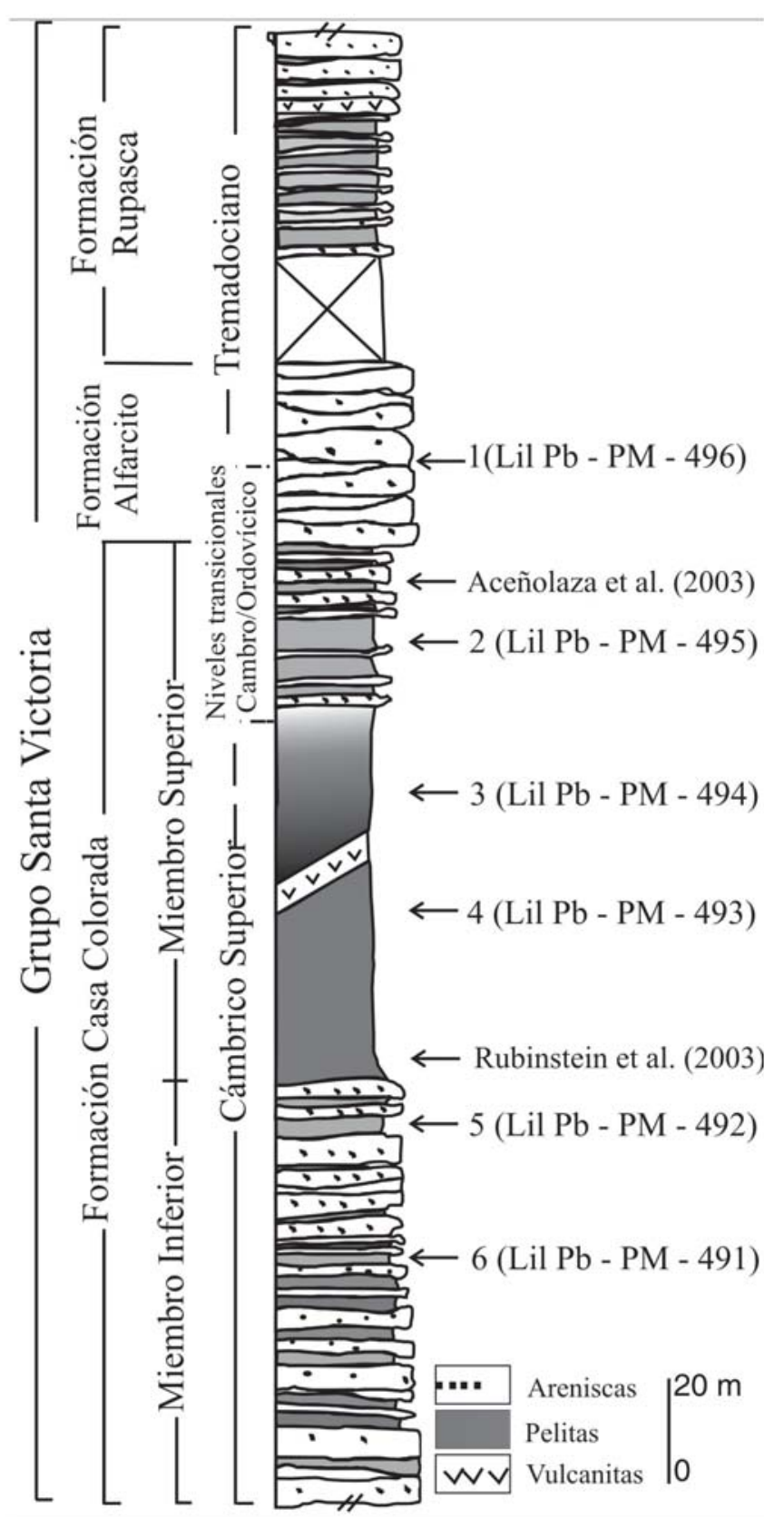

Figura 2. Sección estratigráfica del Grupo Santa Victoria en Quebrada de Moya con ubicación de las muestras palinológicas indicada por los numeros 1-6.

Figure 2. Stratigraphic section of the Santa Victoria Group at the Quebrada de Moya with location of the palynological samples indicated by the numbers $1-6$. 
abundante que la anterior, con la aparición de formas más complejas de los grupos acantomorfos y herkomorfos. Las formas reconocidas corresponden a: Acanthodiacrodium sp., Cristallinium ovillense, Cristallinium randomense, Cymatiogalea velifera, Dactylofusa velifera, Leiosphaeridia sp., Lophosphaeridium spp., Polygonium dentatum, Pterospermella sp., Saharidia fragilis, Timofeevia cf. T. microretis, Vulcanisphaera africana y $V$. turbata. La mayoría de estos taxa reconocidos ocurren en el Cámbrico Tardío, aunque varios de ellos cruzan el límite sistémico y pueden también interpretarse como formas transicionales hasta el Ordovícico más temprano (Figura 3). No obstante, la baja diversidad entre las formas reconocidas y la ausencia de formas del Tremadociano justifican asignar una antigüedad cámbrica tardía para estos niveles. Además, la presencia de especies comunes (Leiosphaeridia sp., Saharidia fragilis, Vulcanisphaera africana) con la asociación descripta por Rubinstein et al.(2003) indicaría cierta correspondencia entre las asociaciones y reforzaría la edad aquí asignada, principalmente basada en los taxa de segura antigüedad cámbrica como Granomarginata squamacea y Celtiberium sp., registrados por Rubinstein et al. (2003) para la asociación por ellos estudiada, proveniente de niveles inferiores próximos (Figura 2).

DISTRIBUCIÓN ESTRATIGRÁFICA DE TAXA SELECCIONADOS

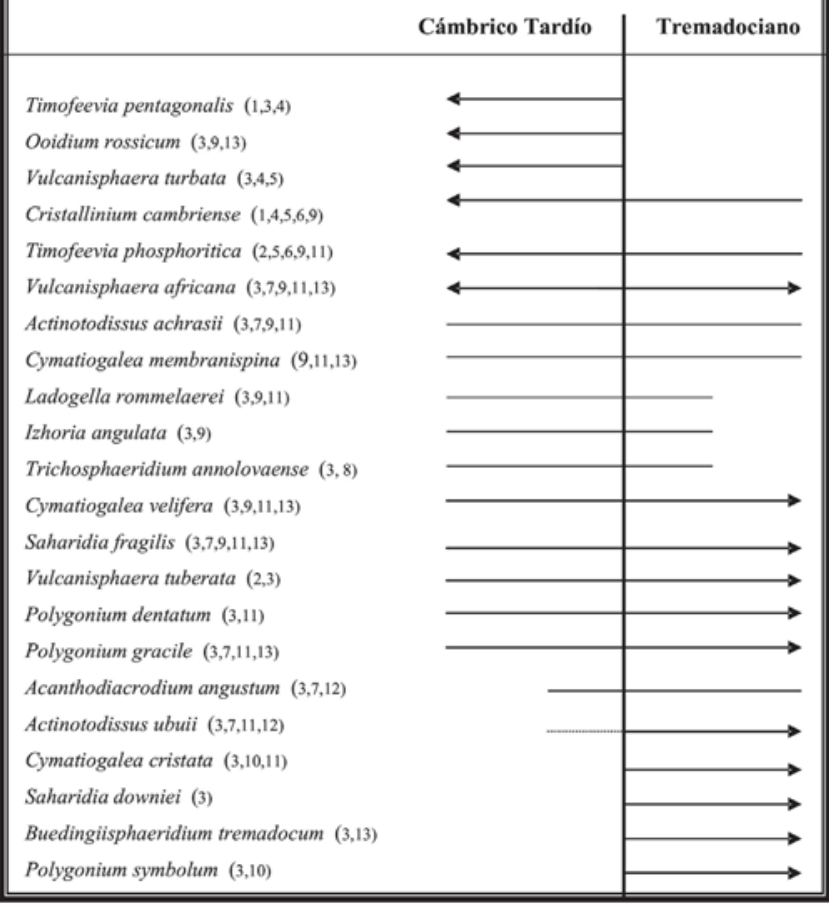

Figura 3. Rango bioestratigráfico de especies seleccionadas presentes en Quebrada de Moya, tomado de registros mundiales previos. (1) Bagnoli, Stouge \& Tongiorgi (1988); (2) Di Milia \& Tongiorgi (1993); (3) Fensome et al. (1990); (4) Martin \& Dean (1981); (5) Martin \& Dean (1988); (6) Moczydlowska \& Crimes, (1995); (7) Moczydlowska \& Stockfors (2004); (8) Paalits \& Heuse (1996); (9) Parsons \& Anderson (2000); (10) Rasul (1979); (11) Vecoli (1996); (12) Vecoli (1999); (13) Vecoli \& Le Hérissé (2004). Figure 3. Biostratigraphic range of selected species present in Quebrada de Moya, considering its previous records.
Una tercera asociación fue recuperada en el nivel superior de la Formación Casa Colorada y en la parte media de la Formación Altarcito, muestras 2 (LIL PB-PM-495) y 1(LIL PBPM-496) en Figura 2. Esta asociación evidencia un cambio significativo con respecto a las inferiores; entre estos cambios está la conspicua diversificación genérica y específica, demostrada por la aparición de grupos diacromorfos y poligonomorfos que se agregan a los ya presentes en niveles inferiores (Figura 4). Es marcado el aumento cuantitativo de especimenes y la mejor preservación de los mismos, como también la aparición de formas típicas del Tremadociano (Acanthodiacrodium angustum, Buedingiisphaeridium tremadocum, Cymatiogalea membranispina, Polygonium symbolum). Estas variables que marcan una importante

\begin{tabular}{|c|c|c|c|}
\hline Asociaciones & 1 & 2 & 3 \\
\hline Espécies /Lil Pb (PM) $\mathrm{N}^{\circ}$ & $491-492$ & $493-494$ & $495-496$ \\
\hline $\begin{array}{l}\text { Ooidium rossicum } \\
\text { cf. Orthosphaeridium? extensum } \\
\text { Cristallinium cambriense } \\
\text { Lophosphaeridium spp. } \\
\text { Leiosphaeridia spp. } \\
\text { Veryhachium sp. } \\
\text { Leiofusa sp. } \\
\text { Timofeevia phosphoritica } \\
\text { Eliasum sp. } \\
\text { Cristallinium randomense } \\
\text { Cristallinium ovillense } \\
\text { cf. Elenia? armillata } \\
\text { Acanthodiacrodium sp. } \\
\text { Cymatiogalea velifera } \\
\text { Timofeevia cf. T. microretis } \\
\text { Stelliferidium sp. } \\
\text { Saharidia fragilis } \\
\text { Vulcanisphaera turbata } \\
\text { Vulcanisphaera africana } \\
\text { Vulcanisphaera tuberata } \\
\text { Ladogella romelaerei } \\
\text { Polygonium dentatum } \\
\text { Vulcanisphaera capillata } \\
\text { Micrhrystridium sp. } \\
\text { Izhoria angulata } \\
\text { Timofeevia pentagonalis } \\
\text { Acanthodiacrodium angustum } \\
\text { Buedingiisphaeridium tremadocum } \\
\text { Cymatiogalea membranispina } \\
\text { Polygonium symbolum } \\
\text { Cymatiogalea velifera } \\
\text { Cymatiogalea cristata } \\
\text { Actinotodissus achrasii } \\
\text { Trichosphaeridium annolovaense } \\
\text { Trichosphaeridium sp. } \\
\text { Actinotodissus ubuii } \\
\text { Polygonium gracile } \\
\text { Impluviculus sp. } \\
\text { Nellia? longispinata } \\
\text { Saharidia downiei } \\
\text { Dasydiacrodium sp. } \\
\text { Actinotodissus crassus } \\
\text { Escolecodonte indet. } \\
\text { Elang }\end{array}$ & $\begin{array}{l}x \\
x \\
x \\
x \\
x \\
x \\
x\end{array}$ & $\begin{array}{l}x \\
x \\
x \\
x \\
x \\
x \\
x \\
x \\
x \\
x \\
x \\
x \\
x \\
x \\
x \\
x \\
x \\
x \\
x\end{array}$ & $\begin{array}{l}x \\
x \\
x \\
x \\
x \\
x \\
x \\
x \\
x \\
x \\
x \\
x \\
x \\
x \\
x \\
x \\
x \\
x \\
x \\
x \\
x \\
x \\
x \\
x \\
x \\
x \\
x \\
x \\
x\end{array}$ \\
\hline
\end{tabular}

Figura 4. Distribución de las especies reconocidas en cada una de las tres asociaciones identificadas.

Figure 4. Distribution of species recognized in each one of the three identified assemblages. 


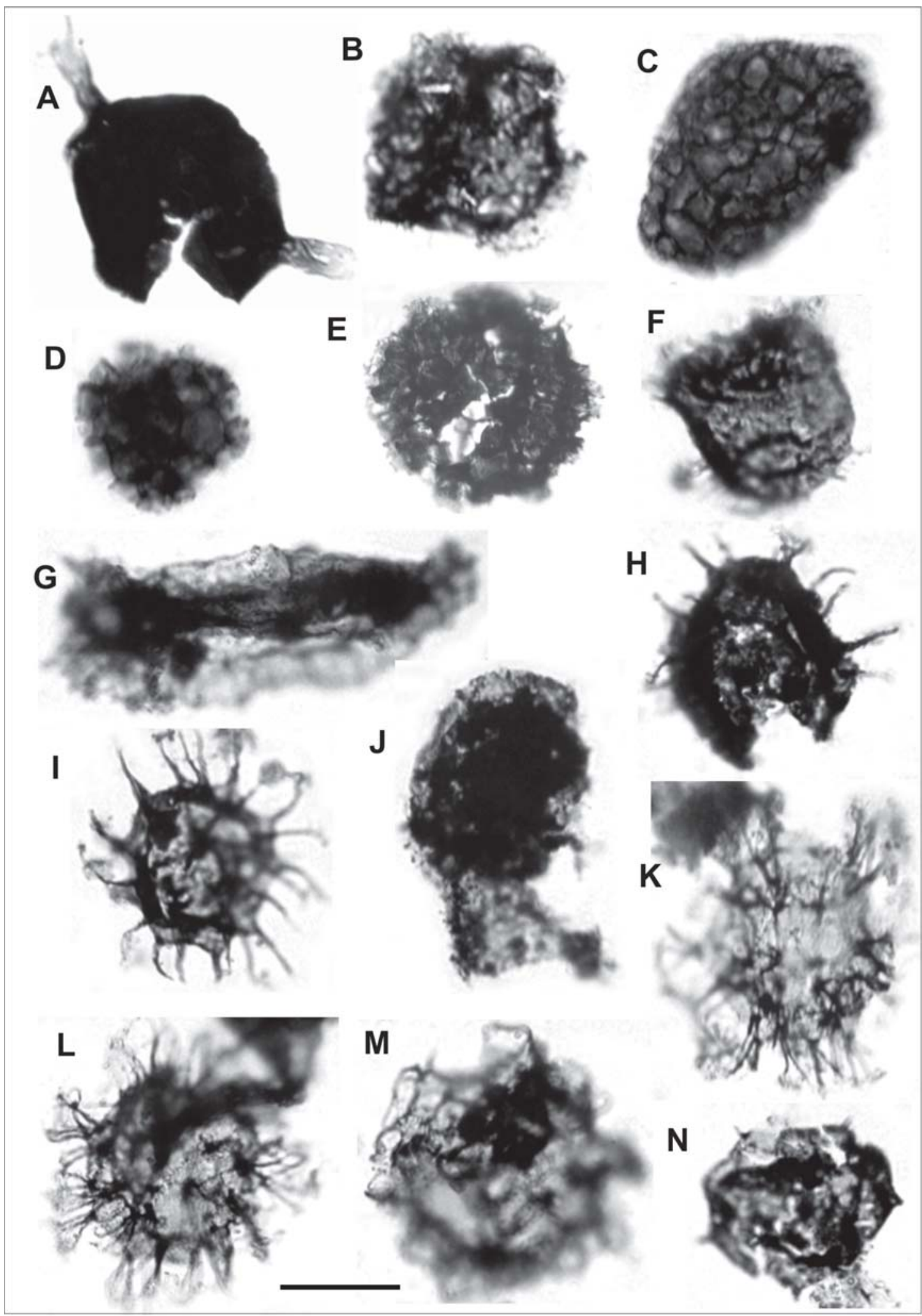

Figura 5. Especies seleccionadas de la sucesión cambro-ordovícica en Quebrada de Moya. Los especimenes están identificados por el número de muestra, preparado y coordenadas del England Finder. A, cf. Orthosphaeridium? extensum (Lil Pb-PM-492-1, EF:56D2); B, Ooidium rossicum (Lil Pb-PM-492-1, EF:58J2); C, Cristallinium ovillense (Lil Pb-PM-493-3, EF:42W1); D, Cristallinium cambriense (Lil Pb-PM-494-1, EF:52C3); E, Cristallinium randomense (Lil Pb-PM-493-2, EF:41M1); F, Ladogella sp., (Lil Pb-PM-494-1, EF:53Y4); G, Dactylofusa velifera (Lil Pb-PM-494-2, EF:53S4); H, Timofeevia phosphoritica (Lil Pb-PM-493-2, EF:36F1); I, Polygonium dentatum (Lil PbPM-493-3, EF:38T); J, cf. Elenia? armillata (Lil Pb-PM-493-2, EF:60H1); K, Timofeevia cf. T. microretis (Lil Pb-PM-493-3, EF:37R2); L, Vulcanisphaera africana (Lil Pb-PM-493-2, EF:26Y2); M, V. turbata (Lil Pb-PM-493-3, EF:52Z4); N, V. tuberata (Lil Pb-PM-494-2, EF:62F2). Escala $=10 \mu \mathrm{m}$.

Figure 5. Selected species from the Cambro-Ordovician succession in the Quebrada de Moya. Specimens are identified by sample and slide number, and England Finder coordinates. Scale bar $=10 \mu \mathrm{m}$. 


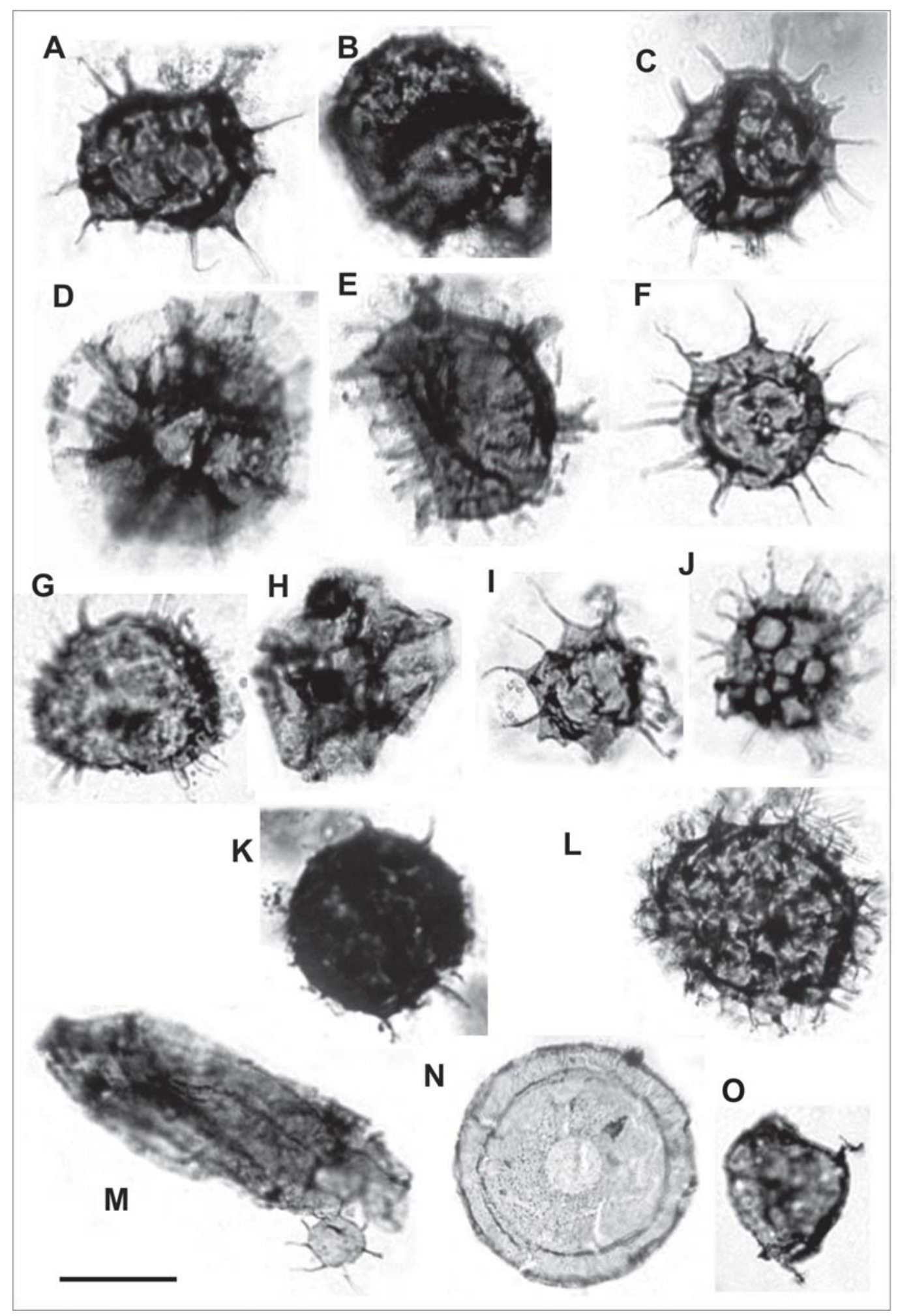

Figura 6. Especies seleccionadas de la sucesión cambro-ordovícica en Quebrada de Moya. Los especimenes están identificados por el número de muestra, preparado y coordenadas del England Finder. A, Actinotodissus achrassi (Lil Pb-PM-496-2, EF:28L1); B, Acanthodiacrodium angustum (Lil Pb-PM-496-2, EF:39K); C, Actinotodissus crassus (Lil Pb-PM-496-1, EF:32B4); D, Cymatiogalea velifera (Lil Pb-PM-495-1, EF:25S4); E, C. cristata (Lil Pb-PM-495-3, EF:22D); F, Dasydiacrodium sp. (Lil Pb-PM-496-1, EF:57E4); G, Ladogella rommelaerei (Lil Pb-PM-496-1, EF:28P4); H, Izhoria angulata (Lil Pb-PM-495-3, EF:53Z2); I, Polygonium gracile (Lil Pb-PM-496-3, EF:42K4); J, P. symbolum (Lil Pb-PM-496-1, EF:36X); K, Timofeevia phosphoritica (Lil Pb-PM-496-1, EF:22R1); L, Stelliferidium sp. (Lil Pb - PM-4951, EF:40X3); M, Eliasum sp.- Impluviculus sp. (Lil Pb-PM-495-1, EF:42B2); N, Saharidia fragilis (Lil Pb-PM-495-1, EF:22C2); O, Veryhachium sp. (Lil Pb-PM-493-1, EF:58N3). Escala $=10 \mu \mathrm{m}$.

Figure 6. Selected species from the Cambro-Ordovician succession in the Quebrada de Moya. Specimens are identified by sample and slide number, and England Finder coordinates. Scale bar $=10 \mu \mathrm{m}$. 
diferencia con niveles inferiores, son tenidas en cuenta para las inferencias e interpretaciones en relación a la ubicación estratigráfica del nivel portador. Por un lado, el grupo de los diacromorfos, que incluye el género Acanthodiacrodium, aunque escasos en el Cámbrico Tardío, son abundantes y extremadamente diversificados durante el Ordovícico Temprano.

Según Vecoli \& Le Hérissé (2004), entre los más importantes bio-eventos producidos en los acritarcos cambro-ordovícicos, están los cortos períodos de diversificación como ocurrió entre lo más tardío del Cámbrico y lo más temprano del Tremadociano, un hecho verificado por la introducción de innovaciones morfológicas de los taxa y un marcado incremento en la diversidad a partir del Ordovícico basal.

Estos argumentos, sumados a la carencia de formas exclusivas del Cámbrico Tardío, con la excepción de los especimenes del género Eliasum, considerados probable redepósito producido por efectos transgresivos, permiten inferir una edad tremadociana para la sección superior de la Formación Casa Colorada y parte media de la Formación Alfarcito, coincidente a los datos aportados previamente en Aceñolaza et al. (2003). Además, entre las dos últimas asociaciones se hallaría el intervalo palinológico de transición cambro-ordovícica, reconocido a nivel mundial por el aumento en la diversidad de los morfotipos, y especiación entre los acritarcos herkomorfos, diacromorfos y poligonomorfos.

Por otro lado, la existencia de una asociación más diversificada y de mejor preservación que las de niveles basales de la sección de Quebrada de Moya sería también coincidente con las interpretaciones sedimentológicas vinculadas al ambiente de depositación. Un aumento en la diversificación con formas más complejas como los géneros Cymatiogalea, Polygonium, Stelliferidium o Vulcanisphaera, serían indicadores de ambientes algo alejados de la playa (offshore) en condiciones de mar abierto y aguas más profundas, coincidente con las interpretaciones determinadas por sus facies sedimentarias. La buena preservación también implica condiciones de mar abierto, mientras que las pobremente preservadas como las asociaciones basales de Quebrada de Moya, son comunes en facies marginales, con excepciones que podrían estar relacionadas por la influencia de paleocorrientes (Vecoli, 2000).

\section{CONCLUSIONES}

Los resultados obtenidos del análisis palinológico de la sucesión del Grupo Santa Victoria en Quebrada de Moya, confirman la antigüedad Cámbrico Tardío-Ordovícico Temprano registrada por otros grupos fósiles. No obstante, se restringe exclusivamente al Cámbrico Tardío el tramo inferior de la Formación Casa Colorada (Miembro Tilcara), mientras que el sector superior de la unidad es asignado al Tremadociano más bajo, junto a la suprayacente Formación Alfarcito en el perfil de Quebrada de Moya. Además, se indica como nivel palinológico de transición sistémico en este perfil, al comprendido a partir del tramo superior de la Formación Casa Colorada (facies heterolíticas del sector superior de la unidad) y hasta la sección inferior de la Formación Alfarcito. También se infieren condiciones paleoambientales marinas poco profundas, en un complejo que registra el pasaje vertical de ambientes proximales a otros más alejados de la playa, bajo condiciones de mar abierto y aguas más profundas, concordante a los datos sedimentológicos (shoreface transicional a offshore inferior).

\section{AGRADECIMIENTOS}

Los autores agradecen a Guillermo Aceñolaza por la información bioestratigráfica aportada y su asistencia durante la realización de esta investigación. También se agradece a los árbitros, Guillermo Ottone y Norma María da Costa Cruz, por el análisis crítico al manuscrito y valiosas sugerencias. Este trabajo fue financiado por CIUNT (Proyecto 26G/230). Se agradece a la Fundación Miguel Lillo por facilitar el uso del Laboratorio de Micropaleontología.

\section{REFERENCIAS}

Aceñolaza, G.F. 1996. Bioestratigrafía del límite CámbricoOrdovícico y Ordovícico basal en la Quebrada de Humahuaca, provincia de Jujuy, República Argentina. Facultad de Ciencias Naturales e IML, Universidad Nacional de Tucumán, Tesis Doctoral, $245 \mathrm{p}$.

Aceñolaza, G.F. \& Albanesi, G.L. 1997. Conodont, trilobite biostratigraphy of the Santa Rosita Formation (Tremadoc) from Chucalezna, Cordillera Oriental, Northern Argentina. Ameghiniana, 34(1):113.

Aceñolaza, G.F.; Aráoz, L.; Poiré, D.G.; Vergel, M.M. \& Albanesi, G. 2003. Biostratigraphical and sedimentological aspects of the Cambro-Ordovician strata at the Angosto de Chucalezna: new palynological data (Jujuy, NW Argentina). Correlación Geológica INSUGEO, Serie Miscelánea, 9:13-21.

Aceñolaza, G.F. \& Poiré, D. 1999. Trace fossils and sedimentology of Rupasca Formation (Lower Ordovician) in Chucalezna, Jujuy province, northern Argentina. Acta Universitatis CarolinaeGeologica, 43(1/2):159-162.

Buatois, L.A. \& Mángano, M.G. 2003. Sedimentary facies and depositional evolution of the Upper Cambrian-Lower Ordovician Santa Rosita Formation in northwest Argentina. Journal of South American Earth Sciences, 16:343-363.

Di Cunzolo, S.; Aceñolaza, G.F. \& Rodríguez Brizuela, R. 2003. Cruziana-Skolithus ichnoassociation in the Casa Colorada Formation (Upper Cambrian-Tremadocian), Cordillera Oriental of Jujuy province, NW Argentina. In: G.L. Albanesi; M.S. Beresi \& S.H. Peralta (eds.) Ordovician from the Andes, INSUGEO, p. 285-288.

Di Milia, A. \& Tongiorgi, M. 1993. Tremadocian acritarch assemblages from the Solanas Sandstone Formation (Nappe Zone of Central Sardinia). Memorie della Società Geologica Italiana, 49:193-204.

Fensome, R.A.; Williams, G.L.; Bars, M.S.; Freeman, J.M. \& Hill, J.M. 1990. Acritarchs and fossil prasinophytes: an index to genera, species and intraspecific taxa. AASP, Contribution Series Number, 25:1-771.

Harrington, H.J. 1957. Ordovician Formations of Argentina. In: HJ. Harrington \& A.F. Leanza (eds.) Ordovician trilobites of Argentina, University of Kansas Press, p. 1-39.

López, C.R. \& Nullo, F.E. 1969. Geología de la margen izquierda de la Quebrada de Humahuaca, de Huacalera a Maimará, Depar- 
tamento Tilcara, provincia de Jujuy, República Argentina. Revista de la Asociación Paleontológica Argentina, 24(3):173-182.

Martin, F. \& Dean, W.T. 1981. Middle and Upper Cambrian and Lower Ordovician acritarchs from Random Island, Eastern Newfoundland. Geological Survey, 343:1-43.

Martin, F. \& Dean, W.T. 1988. Middle and Upper Cambrian acritarchs and trilobite zonation at Manuels River and Random Island, Eastern Newfoundland. Geological Survey, 381:1-91.

Moczydlowska, M. \& Crimes, T.P. 1995. Late Cambrian acritarchs and their age constraints on an Ediacara-type fauna from the Booley Bay Formation, Co. Wexford, Eire. Geological Journal, 30:11-128.

Moczydlowska, M. \& Stockfors, M. 2004. Acritarchs from the Cambrian-Ordovician boundary interval on Kolguev Island, Arctic Russia. Palynology, 28:15-73.

Moya, M.C. 1988. Lower Ordovician in the Southhern part of the Argentine Eastern Cordillera. In: H. Bahlburg; Ch. Breitkreuz \& P. Giese (eds.) The Southern Central Andes, Springer, p. 55-69.

Moya, M.C. 1997. The Iruya unconformity in the Andes of northwestern Argentina: evidence of glaciation during the late Upper Cambrian. In: REGIONAL EUROPEAN MEETING OF SEDIMENTOLOGY, 18, 1997. Abstracts, Heidelberg, 3: 242-243.

Moya, M.C. 1998. El Paleozoico Inferior en la Sierra de Mojotoro, Salta - Jujuy. Revista de la Asociación Geológica Argentina, 53:219-238.

Moya, M.C. 1999. El Ordovícico en los Andes del Norte Argentino. In: G.G. Bonorino; R. Omarini \& J. Viramonte (eds.) Relatorio XIV Congreso Geológico Argentino, Geología del Noroeste Argentino, AGA, 1:134-152.

Moya, M.C. 2002. The Ordovician basin of northern Argentina. In: F.G. Aceñolaza (ed.) Aspects of the Ordovician System in Argentina, INSUGEO, p. 281-294.

Paalits, I. \& Heuse, T. 1996. Taxonomic discusión of the genus Trichosphaeridium Timofeev 1966 and related genera. Acta Universitatis Carolinae-Geologica, 40(3/4):559-575.

Parsons, M.G. \& Anderson, M. 2000. Acritarch microfloral succession from the late Cambrian and Ordovician (early Tremadoc) of Random Island, Eastern Newfoundland, and its comparison to coeval microfloras, particularly those of the East European Platform. AASP, Contribution Series, 38:1-90.

Rasul, S. 1979. Acritarch zonation of the Tremadoc Series of the Shineton Shales, Wrekin, Shropshire, England. Palynology, 3:53-72.

Rubinstein, C.V.; Mángano, M.G. \& Buatois, L.A. 2003. Late Cambrian acritarchs from the santa Rosita Formation: Implications for the Cambrian-Ordovician Boundary in the Eastern Cordillera, northwest Argentina. Revista Brasileira de Paleontologia, 6:43-48.

Tongiorgi, M.; Yin, L.M. \& Stouge, S. 1998. Acritarch and conodonts biofacies reveal sea level changes on Yangtze Plataform during Arenigian times, Dawan Formation, South China. In: SYMPOSIUM COMMISSION INTERNATIONALE DE MICROFLORE DU PALEOZOIQUE, 1, 1998. Abstracts, Pisa, p. 28.

Turner, J.C.M. 1960. Estratigrafía de la Sierra de Santa Victoria y adyacencias. Boletín de la Academia Nacional de Ciencias de Córdoba, 41:163-196.

Vecoli, M. 1996. Stratigraphic significance of acritarchs in CambroOrdovician boundary strata, Hassi-Rmel area, Algerian Sahara. Bolletino della Societa Paleontologica Italiana, 35(1):3-58.

Vecoli, M. 1999. Cambro-Ordovician palynostratigraphy (acritarchs and prasinophytes) of the Hassi-R'Mel area and northern Rhadames Basin, North Africa. Palaeontographia Italica, 86:1-112.

Vecoli, M. 2000. Palaenvironmental interpretation of microphytoplankton diversity trends in the Cambrian-
Ordovician of the northen Sahara Platform, Palaeogeography, Paleoclimatology and Palaeocology, 160:329-346.

Vecoli, M. 2000. Palaenvironmental interpretation of microphytoplankton diversity trends in the CambrianOrdovician of the northen Sahara Platform. Palaeogeography, Paleoclimatology and Palaeocology, 160:329-346.

Vecoli, M. \& Le Hérissé, A. 2004. Biostratigraphy, taxonomic diversity and patterns of morphological evolution of Ordovician acritarchs (organic-walled microphytoplankton) from the northern Gondwana margin in relation to palaeoclimatic and palaeogeographic changes. Earth-Science Reviews, 67:267-311.

Zeballo, F.J.; Albanesi, G.L.; Ortega, G. \& Tortello, F. 2003. Biostratigraphy of Ordovician sequences from Alfarcito area, Tilcara, Eastern Cordillera of Jujuy, Argentina. In: G.L. Albanesi; M.S. Beresi \& S.H. Peralte (eds.) Ordovician from the Andes, INSUGEO, Serie Correlación Geológica, 17:167-171.

Received in January, 2006; accepted in March, 2006.

Apéndice. Lista de taxa de acritarcos y otros palinomorfos citados en el trabajo.

Appendix. List of acritarchs and other palynomorphs cited in the text.

Acanthodiacrodium angustum (Downie) Combaz 1967

Acanthodiacrodium sp.

Actinotodissus achrasii (Martin) Yin 1986

Actinotodissus crassus (Loeblich \& Tappan) Vecoli 1999

Actinotodissus ubuii (Martin) Fensome et al. 1990

Buedingiisphaeridium tremadocum Rasul 1979

Cristallinium cambriense (Slaviková) Vanguestaine 1978

Cristallinium ovíllense (Cramer \& Diez) Fensome et al. 1990

Cristallinium randomense (Martín) Fensome et al. 1990

Cymatiogalea cristata (Downie) Rauscher 1973

Cymatiogalea membranispina Deunff 1961

Cymatiogalea velifera (Downie) Martín 1969

Dactylofusa velifera Cocchio 1982

Dasydiacrodium sp.

cf. Elenia? armillata (Fandeflit) Volkova 1984

Eliasum sp.

Impluviculus sp.

Izhoria angulata Golub \& Volkova 1985

Ladogella rommelaerei (Martín) Di Milia et al. 1989

Ladogella sp.

Leiofusa sp.

Leiosphaeridia spp.

Lophosphaeridium spp.

Micrhrystridium sp.

Nellia? longispinata Parsons \& Anderson 2000

Ooidium rossicum Timofeev 1957

cf. Orthosphaeridium? extensum

Polygonium dentatum (Timofeev ex Konzalová-Mazancová) Albani 1989

Polygonium gracile (Vavrdová) Jacobson \& Achab 1985

Polygonium symbolum Rasul 1979

Saharidia downiei Combaz 1967

Saharidia fragilis (Downie) Combaz 1967

Stelliferidium $\mathrm{sp}$.

Timofeevia cf. T. microretis Martín 1981

Timofeevia pentagonalis (Vanguestaine) Vanguestaine 1978

Timofeevia phosphoritica Vanguestaine 1978

Trichosphaeridium annolovaense Timofeev 1966

Trichosphaeridium sp.

Veryhachium sp.

Vulcanisphaera africana Deunff 1961

Vulcanisphaera capillata Jardiné et al. 1974

Vulcanisphaera tuberata (Downie) Eisenack et al. 1973

Vulcanisphaera turbata Martin en Martin y Dean 1981 